Received: 16 January 2017

Accepted: 12 June 2017

Published online: 21 July 2017

ENTIFIC REP

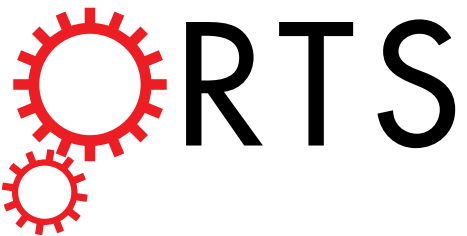

\title{
OPEN Human intrahepatic CD69 + CD8+T cells have a tissue resident memory T cell phenotype with reduced cytolytic capacity
}

Femke Stelma ${ }^{1,2}$, Annikki de Niet ${ }^{1,2}$, Marjan J. Sinnige ${ }^{2}$, Karel A. van Dort ${ }^{2}$, Klaas P. J. M. van Gisbergen ${ }^{3}$, Joanne Verheij ${ }^{4}$, Ester M. M. van Leeuwen ${ }^{2}$, Neeltje A. Kootstra ${ }^{2}$ \& Hendrik W. Reesink $^{1,2}$

Tissue resident memory T cells $\left(T_{R M}\right)$ have been identified in various tissues, however human liver $T_{R M}$ to date remain unidentified. $T_{R M}$ can be recognized by $C D 69$ and/or CD103 expression and may play a role in the pathology of chronic hepatitis $\mathrm{B}$ (CHB) and hepatitis $\mathrm{C}$ virus infection (CHC). Liver and paired blood mononuclear cells from 17 patients (including $4 \mathrm{CHB}$ and $6 \mathrm{CHC}$ patients) were isolated and $\mathrm{CD} 8+\mathrm{T}$ cells were comprehensively analysed by flowcytometry, immunohistochemistry and qPCR. The majority of intrahepatic $C D 8+T$ cells expressed $C D 69$, a marker used to identify $T_{R M}$, of which a subset co-expressed CD103. CD69 + CD8+T cells expressed low levels of S1PR1 and KLF2 and a large proportion (>90\%) was $C X C R 6+$, resembling liver $T_{R M}$ in mice and liver resident NK cells in human. Cytotoxic proteins were only expressed in a small fraction of liver CD69 + CD8 + T cells in patients without viral hepatitis, however, in livers from CHB patients more CD69+CD8+ T cells were granzyme $\mathrm{B}+$. In CHC patients, less intrahepatic CD69+CD8+ T cells were Hobit + as compared to $\mathrm{CHB}$ and control patients. Intrahepatic $\mathrm{CD} 69+\mathrm{CD} 8+\mathrm{T}$ cells likely $\mathrm{T}_{\mathrm{RM}}$ which have a reduced cytolytic potential. In patients with chronic viral hepatitis $\mathrm{T}_{\mathrm{RM}}$ have a distinct phenotype.

The liver is an organ with unique immunologic properties. Generally, a tolerant milieu is maintained in the liver to prevent broad immune activation in response to gut-derived antigens ${ }^{1}$. Hepatotropic viruses, such as hepatitis B (HBV) and C virus (HCV), are therefore thought to specifically target the liver for infection. During an infection, upon encounter with their cognate antigen, antigen-specific $\mathrm{T}$ cells undergo clonal expansion and subsequently form a memory $\mathrm{T}$ cell population ${ }^{2}$. Recently, it became evident that this memory population does not only consist of a recirculating fraction - detectable in the peripheral blood - but also includes an important tissue resident memory $\mathrm{T}$ cell $\left(\mathrm{T}_{\mathrm{RM}}\right)$ pool, residing in non-lymphoid organs ${ }^{3}$. $\mathrm{T}_{\mathrm{RM}}$ can be identified by expression of CD69, identifying a broad population of which a subset of cells co-expresses CD103 (integrin alpha $E)^{3,4}$. $T_{R M}$ reside in human tissues such as lung, skin and gut and have unique functions $\mathrm{s}^{3,5-7}$. For example, resident intrahepatic, but not circulatory CD8 + T cells, are the main effectors in an effective immune response against malaria in mice ${ }^{8}$. In viral hepatitis, local bystander $\mathrm{T}_{\mathrm{RM}}$ may play an important role in the pathology observed in chronic viral infection of the liver?

Whereas previous data has identified a large CD69 + NK cell population in the liver ${ }^{10}$, data on CD8+ T cells with a tissue resident phenotype in the human liver is lacking, as is knowledge on their phenotype in patients with viral hepatitis. The aim of this study was to examine the presence of intrahepatic $\mathrm{T}_{\mathrm{RM}}$ in control patients without viral hepatitis, as well as the presence of these cells in the liver from patients who are chronically infected with HBV or HCV.

\footnotetext{
${ }^{1}$ Department of Gastroenterology and Hepatology, Academic Medical Center, Amsterdam, The Netherlands. ${ }^{2}$ Department of Experimental Immunology, Academic Medical Center, Amsterdam, The Netherlands. ${ }^{3}$ Department of Hematopoiesis, Sanquin Research and Landsteiner Laboratory, Academic Medical Center, Amsterdam, Netherlands. ${ }^{4}$ Department of Pathology, Academic Medical Center, Amsterdam, The Netherlands. Neeltje A. Kootstra and Hendrik W. Reesink jointly supervised this work. Correspondence and requests for materials should be addressed to H.W.R. (email: h.w.reesink@amc.nl)
} 
a

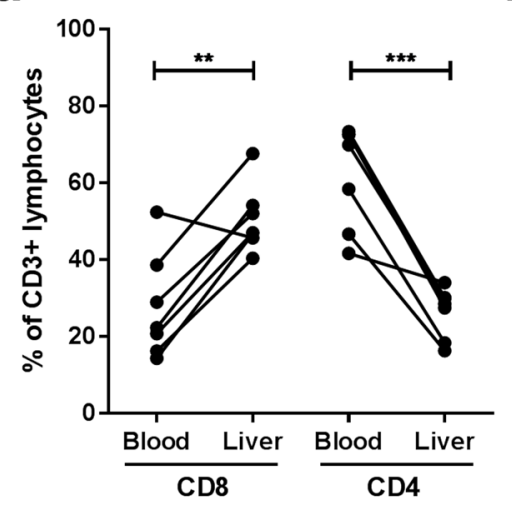

d

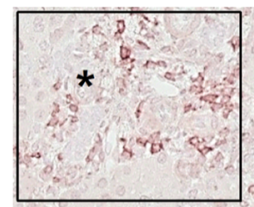

e

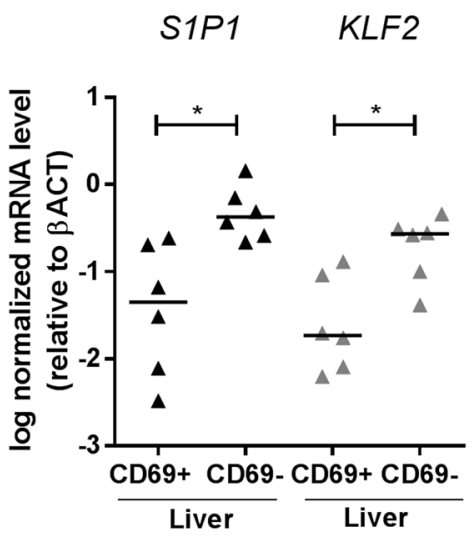

b

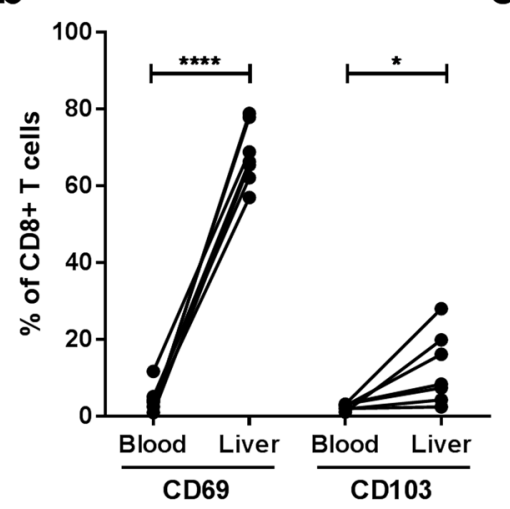

C

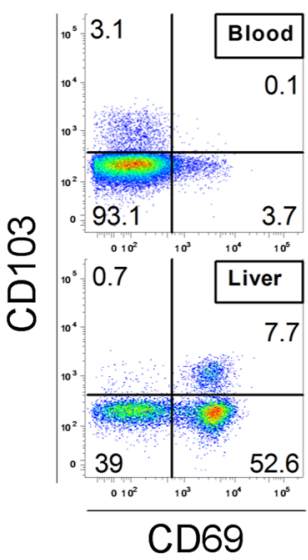

CD69

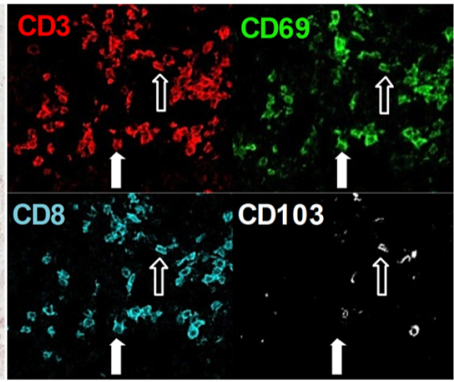

f

CXCR6

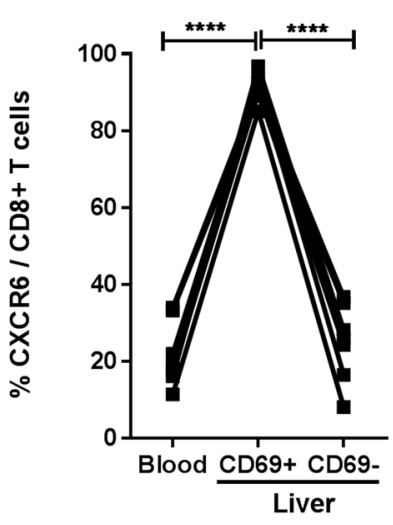

Figure 1. (a) Frequency of CD8 + and CD4+ T cells as a percentage of total CD3 + lymphocytes was compared in control blood and liver $(n=7)$. Statistical analyses; paired t-test. (b) Frequency of CD69+ and CD103+ as a percentage of total CD8 $+\mathrm{T}$ cells in blood and liver. Statistical analyses; paired t-test. (c) Representative flow cytometry plots showing CD69 and CD103 expression, gated on CD8 + CD3 + positive lymphocytes. (d) Paraffin embedded formalin-fixed sections of control livers $(n=3)$ (tumor-free tissue was used) were stained using sequential immunohistochemistry. An immunohistochemical staining of CD69 is shown with a portal field (*) and a central vein (\#), and a portal field with co-localization of CD69+CD8 + CD3+ lymphocytes (filled arrow) and CD69 + CD103 + CD8 + CD3 + lymphocytes (open arrow). Original magnification: 20x. (e) Relative expression of S1PR1 and transcription factor KLF2 in sorted CD69+ and CD69-CD8+ T cells from the liver $(n=6)$. Bars indicate median. Statistical analyses; Wilcoxon signed rank test. (f) Frequency of CXCR6+ T cells as a percentage of total CD8 + T cells in blood, liver CD69+ and liver CD69-CD8+ T cells. Statistical analyses; paired t-test. $* \mathrm{p}<0.05, * * \mathrm{p}<0.01, * * * \mathrm{p}<0.001, * * * * \mathrm{p}<0.0001$.

\section{Results}

Human intrahepatic CD69 + T cells express a tissue resident phenotype. In the liver, CD8+ T cells were enriched as compared to the blood $(\mathrm{p}=0.0042)$. Inversely, the liver contained fewer CD $4+\mathrm{T}$ cells than the blood $\left(p=0.0004\right.$, Fig. 1a). To identify intrahepatic $T_{R M}$, we analysed the expression of two markers 
a

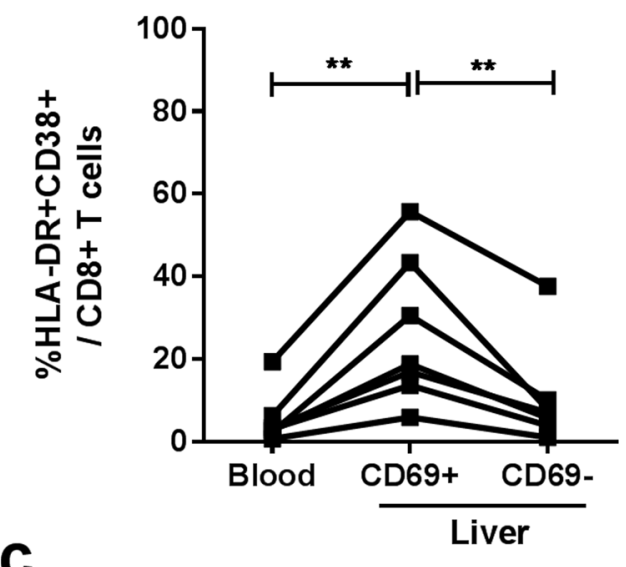

PD-1

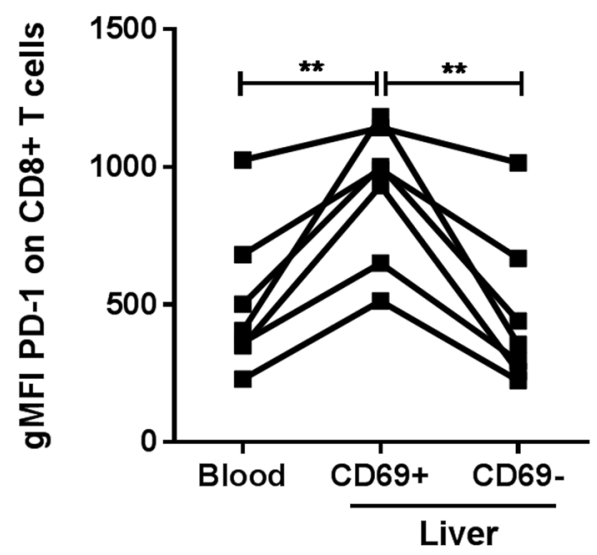

b

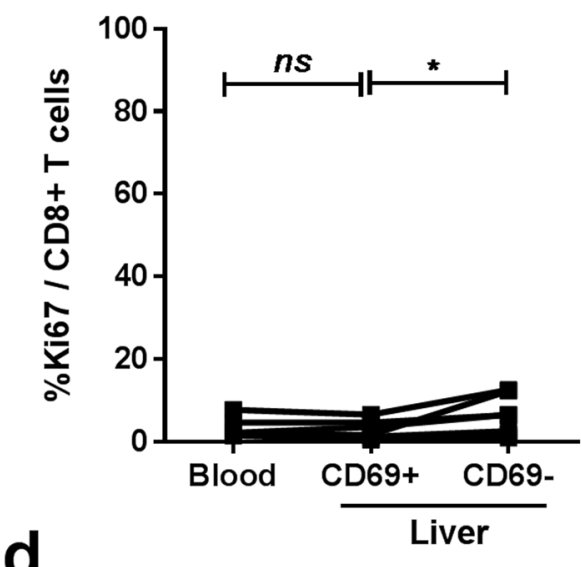

Nur77

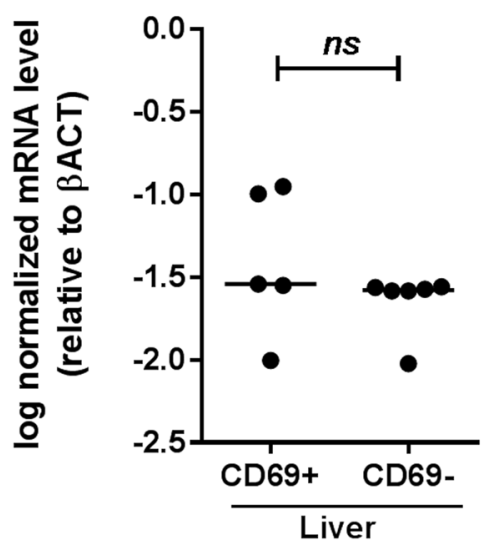

Figure 2. Frequency of HLA-DR $+\mathrm{CD} 38+$ double positive (a) and Ki67 (b) positive cells as a percentage of CD8+ T cells and gMFI of PD-1 (c) in control blood vs. liver (CD69+ and CD69-) CD8+ T cells. Statistical analyses; paired t-test. **p $<0.01$. (d) Relative expression of Nur77 in sorted CD69+ and CD69-CD8+ T cells from the liver $(n=6$, one CD69+ sample was unavailable). Bars indicate median. Statistical analyses; Wilcoxon signed rank test. Abbreviations: ns, non-significant.

commonly expressed by $\mathrm{T}_{\mathrm{RM}}$; $\mathrm{CD} 69$ and $\mathrm{CD}_{103^{5,11}}$. In the blood, few CD69 positive cells were present within the CD8+ T cell population (mean 4.3\%), while a significant population of CD69+CD8+ T cells was detected in the liver (mean $68.0 \%, \mathrm{p}<0.0001$, Fig. 1b, gating strategy in Supplementary Fig. 1). In the peripheral blood, mean $2.5 \%$ of CD8 $+\mathrm{T}$ cells expressed CD103, while mean $12.4 \%$ of intrahepatic CD8+ T cells were CD103 positive $(\mathrm{p}=0.03$, Fig. $1 \mathrm{~b}, \mathrm{c})$. In contrast to those in the blood, the CD103 + CD $8+\mathrm{T}$ cells detected in the liver co-expressed CD69 (Fig. 1c). Immunohistochemistry revealed localization of CD69+ CD8 + CD3 + positive cells in portal fields, central veins, and parenchymal zones 1-3 (Fig. 1d, Supplementary Fig. 2).

The sphingosine 1-phosphate receptor-1 (S1PR1) is crucial for recirculation of (naïe) $\mathrm{T}$ cells ${ }^{12}$, and negatively regulated by $\mathrm{CD} 69^{7,11-13}$. S1PR1 and its regulatory transcription factor Krüppel-like Factor 2 (KLF2) were significantly down-regulated in sorted intrahepatic CD69+ CD8 + T cells as compared to CD69-CD8+ T cells (Fig. 1e). Chemokine receptor CXCR6, associated with homing to the liver ${ }^{10,14,15}$, was expressed by mean $91.2 \%$ of CD69 + CD8 + T cells in the liver, as compared to mean $25.0 \%$ of their CD69- liver counterparts $(\mathrm{p}<0.0001)$, and mean $21.9 \%$ of peripheral blood CD8 + T cells (Fig. 1f). Additionally, $25-35 \%$ of CD69+ CD8 + T cells in the liver were positive for the MR1 tetramer, CD161 and IL-18R $\alpha$, specific for mucosa associated invariant T (MAIT) cells (Supplementary Fig. 3) ${ }^{16}$.

Intrahepatic CD69 + CD8 + T cells are not recently activated. In intrahepatic CD69+CD8+ T cells, the proportion of HLA-DR/CD38 double positive T cells, which in the peripheral blood represent activated cells, was significantly higher (mean $26.4 \%$ ) than in peripheral blood T cells (mean $5.4 \%, p=0.0049$, Fig. 2a, Supplementary Fig. 4). Whereas alanine aminotransferase (ALT) levels significantly correlated with the proportion of HLA-DR/CD38 expressing CD8 $+\mathrm{T}$ cells in the blood $(\mathrm{p}=0.01)$, in the liver there was no correlation between the two ( $p=0.33$, Supplementary Fig. 5). The proportion of Ki67 + cells, which represent cells that have recently divided, was significantly higher in intrahepatic CD69-CD8+ T cells as compared to CD69+ CD8+ T cells (mean $6.5 \%$ and $1.6 \%$ respectively, $p=0.04$, Fig. $2 \mathrm{~b}$ ). The proportion of cells expressing apoptosis marker 
a

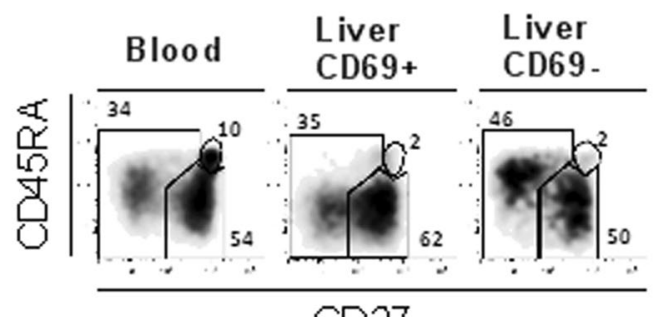

b

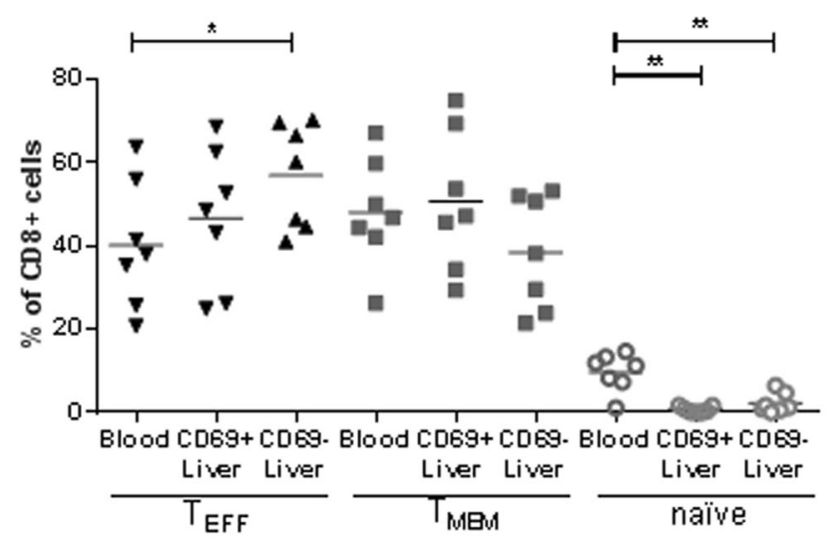

Figure 3. (a) Representative flow cytometry plots showing CD27 and CD45RA expression with gates indicating effector memory $\left(\mathrm{T}_{\mathrm{EFF}}, \mathrm{CD} 45 \mathrm{RA} \pm \mathrm{CD} 27-\right)$, memory $\left(\mathrm{T}_{\mathrm{MEM}}, \mathrm{CD} 45 \mathrm{RA}-\mathrm{CD} 27+\right)$ and naïve $(\mathrm{CD} 45 \mathrm{RA}+\mathrm{CD} 27+)$ CD8 $+\mathrm{T}$ cells in control blood and liver (CD69+ and CD69-). (b) Frequency of $\mathrm{T}_{\mathrm{EFF}}$, $\mathrm{T}_{\mathrm{MEM}}$ and naïve CD8 $+\mathrm{T}$ cells as a percentage of total CD8 $+\mathrm{T}$ lymphocytes in control blood and liver (CD69+ and CD69-). Bars indicate mean. Statistical analyses; paired t-test $* \mathrm{p}<0.05, * * \mathrm{p}<0.01, * * * * \mathrm{p}<0.0001$. Abbreviations: ns, non-significant.

TO-PRO-3, was low in all populations (Supplementary Fig. 6). Programmed death-1 (PD-1), associated with $\mathrm{T}$ cell inhibition, was higher expressed on liver CD69+ CD8 + T cells as compared to liver CD69- cells and peripheral blood $\mathrm{T}$ cells (Fig. 2c). Other inhibitory molecules were not differentially expressed on intrahepatic CD69 + and CD69- CD8+ T cells (Supplementary Fig. 6). Nur77, an 'immediate early gene', up-regulated in response to TCR stimulation ${ }^{17}$, was not differentially expressed in CD69 + and CD69-CD8+ T cells isolated from the liver (Fig. 2d). As expected, the proportion of naïve peripheral blood CD8 $+\mathrm{T}$ cells expressing activation markers was low (Supplementary Fig. 7).

Intrahepatic CD69 + CD8 + T cells are not naïve, but have a memory or effector-memory phenotype, similar to the peripheral blood. CD8 $+\mathrm{T}$ cells can be divided into different subsets, including naive and memory cells, the latter comprising cells that have been primed by antigen. Antigen experienced cells can be divided into memory $\mathrm{T}$ cells $\left(\mathrm{T}_{\mathrm{MEM}}\right)$ and effector-type memory $\mathrm{T}$ cells $\left(\mathrm{T}_{\mathrm{EFF}}\right)$. We identified these populations by CD27 and CD45RA expression, and analysed naïve (CD45RA + CD27+), $\mathrm{T}_{\mathrm{MEM}}$ (CD45RA-CD27+) and $\mathrm{T}_{\mathrm{EFF}}$ (CD45RA \pm CD27-) $\mathrm{T}$ cells (Fig. 3a) ${ }^{2,18,19}$. The proportion of $\mathrm{T}_{\mathrm{EFF}}$ was comparable in blood and intrahepatic CD69 + CD8 + T cells (Fig. 3b). In liver CD69-CD8+ T cells, a significantly higher proportion of CD8+ T cells were $\mathrm{T}_{\mathrm{EFF}}$ phenotype as compared to the blood $(\mathrm{p}=0.01)$. The proportion of $\mathrm{T}$ cells that had a $\mathrm{T}_{\mathrm{MEM}}$ phenotype was not different between blood, intrahepatic CD69+ and intrahepatic CD69- CD8+ T cells. Naïve cells were significantly less frequent in the CD8 + T cell populations derived from the liver (CD69+ and CD69- mean 0.7\%, $2.2 \%$ respectively) as compared to the blood (mean $9.7 \%$, Fig. 3a,b).

The majority of intrahepatic CD69 $+\mathrm{CD} 8+\mathrm{T}_{\mathrm{EFF}}$ cells do not express cytotoxic proteins. As intrahepatic NK cells expressing CD69 and CXCR6 have a reduced cytolytic potential ${ }^{10}$, we next analysed the expression of these effector molecules in intrahepatic $\mathrm{T}_{\mathrm{EFF}}$ and $\mathrm{T}_{\mathrm{MEM}}$ subsets. The proportion of intrahepatic $\mathrm{CD} 69+\mathrm{T}_{\mathrm{EFF}}$ cells expressing perforin was significantly lower (mean 12.1\%) that of blood $\mathrm{T}_{\mathrm{EFF}}$ cells (mean $91.8 \%$, $\mathrm{p}<0.0001$ ) as was the proportion of granzyme $\mathrm{B}+$ cells (mean $45.7 \%$ and 82.4 respectively, $\mathrm{p}=0.0001$, Fig. $4 \mathrm{a}, \mathrm{c}$ ). Within the intrahepatic $\mathrm{T}_{\mathrm{EFF}}$ cell population expressing CD69, significantly less cells expressed cytotoxic proteins as compared to CD69- $\mathrm{T}_{\mathrm{EFF}}$ cells $(\mathrm{p}<0.0001$ for perforin and $\mathrm{p}=0.001$ for granzyme $\mathrm{B})$. In addition, significantly less intrahepatic $\mathrm{T}_{\mathrm{MEM}}$ expressed perforin (CD69+; mean 9.3 and CD69-; mean 19.8\%) than in the blood (mean $52.6 \%, \mathrm{p}=0.0002$, Fig. $4 \mathrm{~b}, \mathrm{c}$ ), whereas no difference in $\mathrm{T}_{\mathrm{MEM}}$ granzyme B expression was observed (Fig. $4 \mathrm{~b}, \mathrm{c}$ ). 

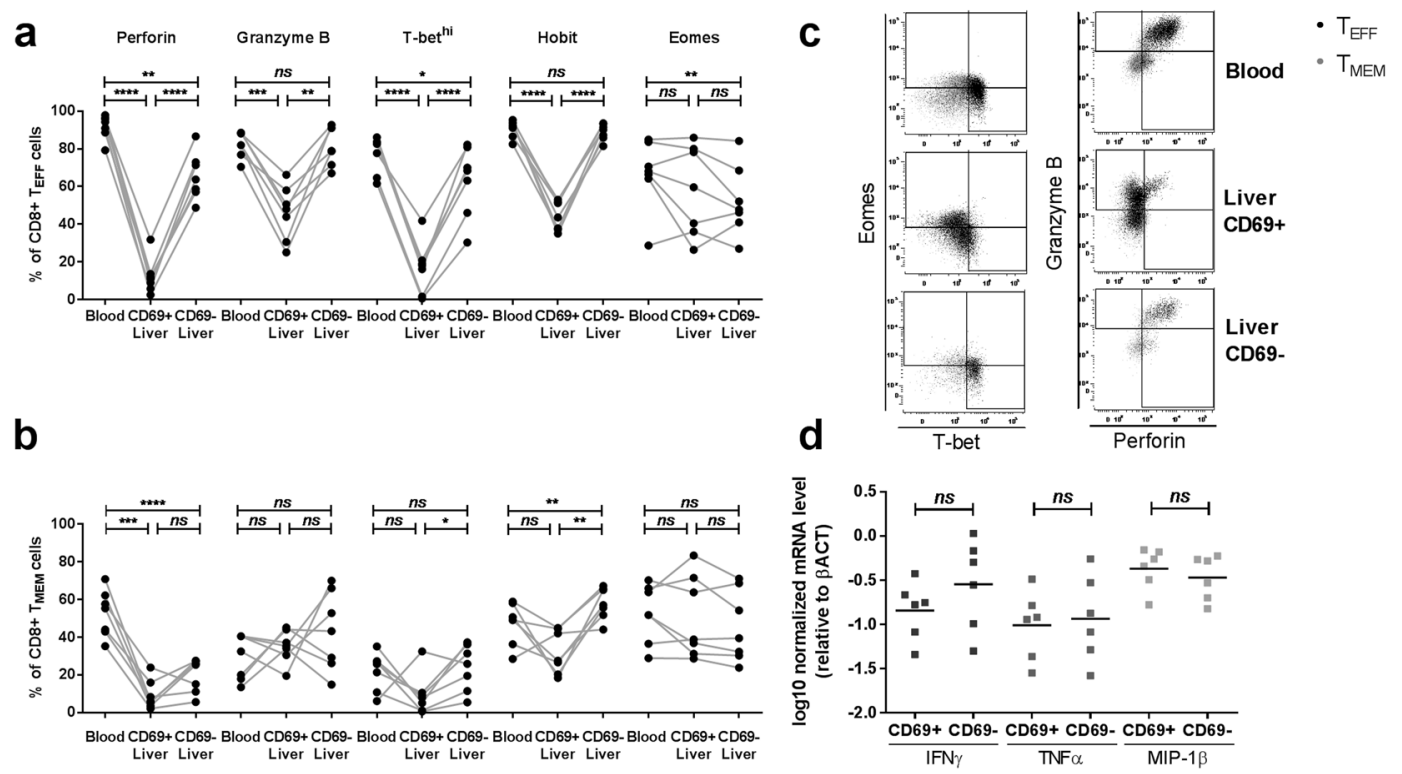

Figure 4. Frequency of perforin + , granzyme B + , Tbet ${ }^{\text {hi }}$, Hobit + and Eomes + cells as a percentage of CD $8+$ $\mathrm{T}_{\mathrm{EFF}}(\mathbf{a})$ and $\mathrm{T}_{\mathrm{MEM}}(\mathbf{b})$ in control blood and liver (CD69+ and CD69-). Statistical analyses; paired t-test. (c) Representative FACS staining and gating for perforin, granzyme B, Eomes and T-bet in blood and liver CD69 + ad CD69- $\mathrm{T}_{\mathrm{EFF}}$ and $\mathrm{T}_{\mathrm{MEM}}$ cells. Statistical analyses; paired t-test. (d) Relative expression of cytokines interferon- $\gamma(\operatorname{IFN} \gamma)$, tumor necrosis factor- $\alpha$ (TNF $\alpha)$ and macrophage inflammatory protein $1 \beta$ (MIP-1 $\beta$ ) in sorted CD69+ and CD69-CD8+ T cells from control livers. Bars indicate median. Statistical analyses; Wilcoxon signed rank test. $* \mathrm{p}<0.05, * * \mathrm{p}<0.01, * * * \mathrm{p}<0.001 * * * * \mathrm{p}<0.0001$. Abbreviations: ns, non-significant.

The majority of CD69 $+C D 8+T_{E F F}$ cells in the liver do not express transcription factors T-bet and Hobit. Memory formation and effector function is tightly regulated by transcription factors such as the T-box transcription factors T-bet, "homolog of Blimpl in T cells" (Hobit) and Eomesodermin (Eomes) ${ }^{20-22}$. In the liver, a significantly less CD69 $+\mathrm{T}_{\mathrm{EFF}}$ cells expressed T-bet (mean 16.9\%) as compared to blood $\mathrm{T}_{\mathrm{EFF}}$ (mean 77\%, $\mathrm{p}<0.0001$, Fig. 4a,c). As in the blood, the majority of intrahepatic CD69- $\mathrm{T}_{\mathrm{EFF}}$ cells expressed T-bet (mean 63\%). Hobit was expressed in $\mathrm{CD} 69+\mathrm{CD} 8+\mathrm{T}_{\mathrm{FFF}}$ from the liver in significantly less cells (mean 44.2\%) than in the blood (mean 90.7\%) and CD69- liver CD8+ T cells (mean 88.9\%, $\mathrm{p}<0.0001$ ). In naïve peripheral blood CD8+ $\mathrm{T}$ cells expression these transcription factors was only observed in a small proportion of cells (mean T-bet 1.94\%, Hobit 5.7\% and Eomes 9.96\%, Supplementary Fig. 7).

Intrahepatic $C D 69+T_{R M}$ express equal amounts of cytokine mRNA as CD69- liver cells. We next investigated the potential of liver-resident T-cells to produce antiviral and proinflammatory cytokines. Cytokine mRNA content was assessed in intrahepatic CD69+ and CD69- cells. No differences were observed in interferon- $\gamma($ IFN- $\gamma)$, tumor necrosis factor- $\alpha$ (TNF- $\alpha$ ) and macrophage inflammatory protein- $1 \beta$ (MIP-1 $\beta$ ) mRNA expression between CD69+ and CD69-CD8+ T cells (Fig. 4d).

Intrahepatic CD69 + CD8 + T cells express a distinct phenotype in chronic viral hepatitis. The proportion of CD8 + T cells in the liver of patients with $\mathrm{HBV}$ and $\mathrm{HCV}$ was similar to that of control patients (Fig. 5a). The proportion of $\mathrm{CD} 4+\mathrm{T}$ cells was significantly higher in the livers of patients with $\mathrm{CHC}$ (median $52.8 \%$ ) as compared to control patients (median $28.5 \%, \mathrm{p}=0.02$, Fig. $5 \mathrm{a}$ ). As in control patients, the majority of CD8 + T cells in the liver of patients with CHB or CHC expressed CD69 (median 66.2\%, 79.9\% and 75.6\%). A minority of these cells co-expressed CD103 and this population was significantly expanded in patients with CHC (median 25.7\%) as compared to control patients (median 8.4\%, $\mathrm{p}=0.03$, Fig. 5b). Similar to control patients, in CHB and CHC patients, most intrahepatic CD69 + CD8+ T cells were CXCR6 positive (Supplementary Fig. 8a). HLA-DR/CD38, Ki67 and PD-1 were similarly expressed on CD69 + CD8+ T cells in CHB, CHC and control patients. In CHB patients, significantly more $\mathrm{CD} 69+\mathrm{CD} 8+\mathrm{T}_{\mathrm{EFF}}$ cells expressed cytotoxic protein granzyme B (median $74.4 \%$ ) as compared to controls (median $48.1 \%, p=0.012$, Fig. $5 \mathrm{~d}$ ), while this was similar between CHC patients and controls. In CHC patients, significantly less CD69+ CD8+ liver T cells expressed Hobit as compared to control CD69+CD8 $+\mathrm{T}$ cells ( $\mathrm{T}_{\mathrm{EFF}}$ as well as $\mathrm{T}_{\mathrm{MEM}}, p=0.0025$, Fig. 5e). These differences between patient groups were not observed in intrahepatic CD8 $+\mathrm{T}$ cells that were CD69 negative (data not shown). The percentage of cells expressing perforin and transcription factors T-bet and Eomes did not differ in patients with CHB and CHC and controls (Fig. 5c, Supplementary Fig. 8b).

\section{Discussion}

In this study we have for the first time in man characterized a population of CD69+CD8+ T cells with a tissue resident-like phenotype which is abundantly present in the liver, but not in the peripheral blood. Expression of CD69 identifies $T_{R M}$ in many different human tissues ${ }^{3,7}$, as well as on the majority of intrahepatic $T_{R M}$ in mice 
a

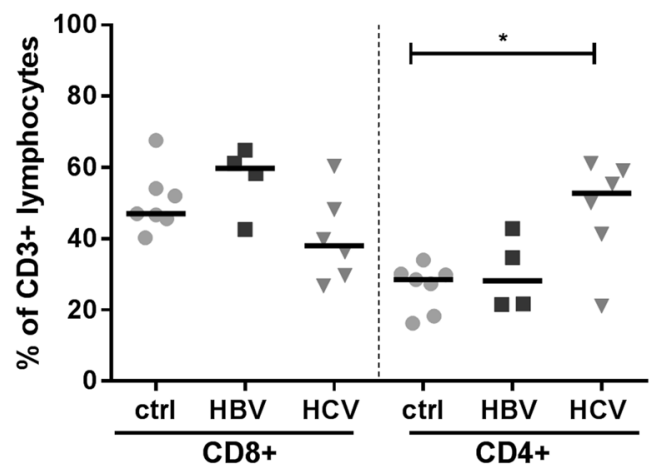

b

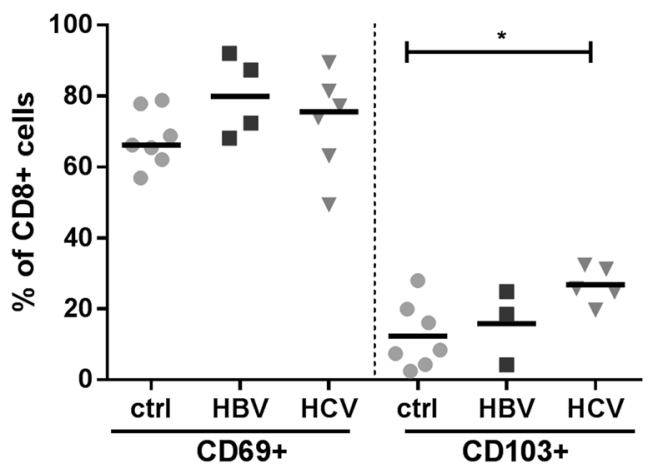

C
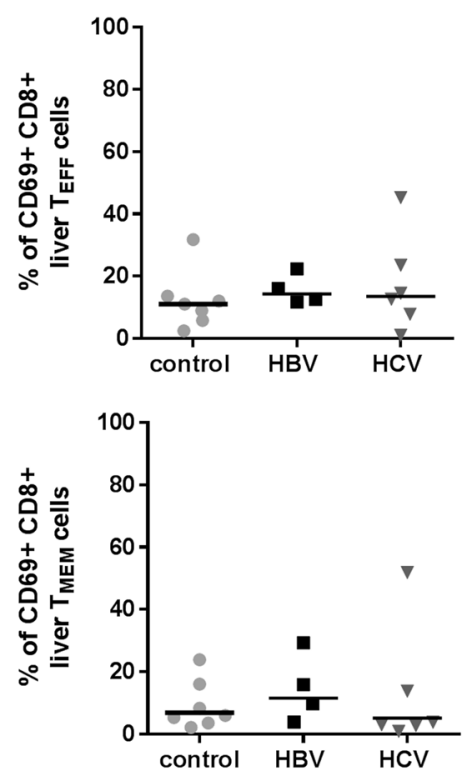

d
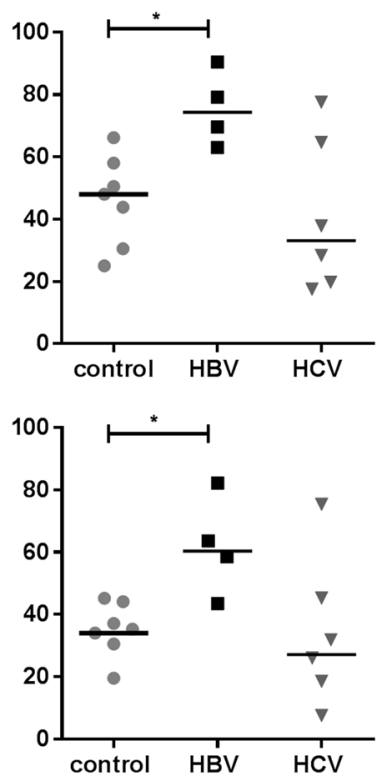

e
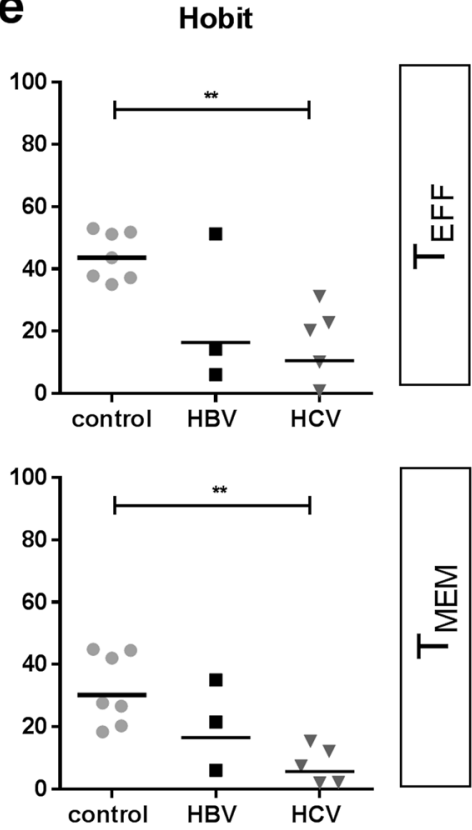

Figure 5. (a) Frequency of CD8 + and $\mathrm{CD} 4+\mathrm{T}$ cells as a percentage of total CD3 $+\mathrm{T}$ lymphocytes in control (ctrl, $\mathrm{n}=7$ ), CHB infected (HBV, $\mathrm{n}=3-4)$ and $\mathrm{CHC}$ infected (HCV, $\mathrm{n}=5-6$ ) livers (number of samples depending on sample availability). Bars indicate median. Statistical analyses; Mann-whitney test. (b) Frequency of CD69+ and CD103+ as a percentage of total CD8+ T lymphocytes in control, HBV and HCV livers. Bars indicate median. Statistical analyses; Mann-whitney test. Frequency of perforin $+(\mathbf{c})$, granzyme $\mathrm{B}+(\mathbf{d})$ and Hobit $+(\mathbf{e})$ CD8 + T cells as a percentage of intrahepatic CD69 + CD8 $+\mathrm{T}_{\mathrm{EFF}}$ (upper graphs) and $\mathrm{T}_{\mathrm{MEM}}$ (lower graphs). Bars indicate median. Statistical analyses; Mann-whitney test. ${ }^{*} \mathrm{p}<0.05, * * \mathrm{p}<0.01$.

and resident-like NK cells in the human liver ${ }^{10,13,23}$. A small proportion of intrahepatic CD69+ CD8 + T cells expressed CD103, in accordance with data on mouse liver $\mathrm{T}_{\mathrm{RM}}{ }^{13}$. Subsets of human $\mathrm{T}_{\mathrm{RM}}$ including those in lung, brain, skin and gut ${ }^{3,24}$ co-express CD103, which forms a heterodimer with integrin $\beta 7$ and recognizes E-cadherin, which is expressed on hepatocytes and bile duct epithelium. In patients with $\mathrm{CHC}$, this population was enriched, possibly causing portal infiltrates and liver pathology ${ }^{25}$. CD69 is known to antagonize the expression of the S1P receptor S1PR $1^{12}$, which can detect an S1P gradient leading out of the tissues ${ }^{11,12,26}$. Lower expression of S1PR1 and its regulatory transcription factor KLF2 in intrahepatic CD69+CD8+ T cells suggests that these cells are not able to egress from the liver, and are indeed $\mathrm{T}_{\mathrm{RM}}$, as described for mouse liver $\mathrm{T}_{\mathrm{RM}}{ }^{27}$. Parabiosis experiments, in which mice share their circulation, revealed that nearly all CD8 + T cells expressing CD69 are non-circulatory and interestingly, these $\mathrm{T}_{\mathrm{RM}}$ could be localized in the parenchyma as well as within large vessels in the liver ${ }^{23}$. Here, immunohistochemistry localized CD69 + CD8 + T cells in the parenchyma as well as within portal fields of the liver. Expression of CXCR6 has been associated with human liver infiltrating lymphocytes ${ }^{14}$, intrahepatic NK cells ${ }^{10}$, and protective responses against malaria in mice ${ }^{15}$. High expression of CXCR6 could facilitate maintenance in the liver as its ligand, CXCL16, is present on hepatocytes and liver sinusoidal endothelial cells (LSEC) in healthy and HCV infected livers ${ }^{14,28}$. Few naïve cells could be identified in the population of CD69+CD8+ 
T cells from the liver, supporting the hypothesis that CD69 identifies a distinct non-circulating population of memory $\mathrm{T}$ cells ${ }^{29,30}$.

CD69 has originally been identified as a marker of recent activation. To investigate the activation state of CD69 + CD8 + T cells, we examined the expression of other markers of recent and chronic stimulation. Low numbers of blood and liver CD8 $+\mathrm{T}$ cells were Ki67 positive, suggesting the absence of persistent $\mathrm{T}$ cell activation as observed in malaria specific intrahepatic $\mathrm{T}_{\mathrm{RM}}{ }^{27}$. Nur77, a factor upregulated after recent TCR activation ${ }^{17}$, was not differentially expressed in CD69+ and CD69-CD8+ T cells from the liver, suggesting that the expression of CD69 was not promoted by recent TCR cross-linking. CD69+ CD8+ T cells in the liver contained a higher fraction of HLA-DR/CD38 double positive cells, which is consistent with data regarding lung residing CD8 $+\mathrm{T}$ cells $^{31}$. The proportion of HLA-DR expressing cells correlated with ALT in the peripheral blood but not in the liver, suggesting in the liver immune activation is not the primary mechanism of HLA-DR/CD38 upregulation. In addition, CD69 + CD8 + T cells expressed PD-1 at higher levels than CD69- liver CD8+ T cells and blood CD8+ $T$ cells which could play a role in maintaining local tolerance, similar to what has been reported for CD103 $+\mathrm{T}_{\mathrm{RM}}$ in the brain ${ }^{32}$. Local induction of apoptosis to induce tolerance did not seem to play a role in the liver ${ }^{33}$.

A hallmark of effector type CD8 + T cells in the periphery is the presence of intracellular cytotoxic proteins, such as perforin and granzyme $\mathrm{B}^{2}$. Significantly less CD69+CD8 $+\mathrm{T}_{\mathrm{EFF}}$ cells in the liver expressed cytotoxic proteins, as compared to peripheral effector cells, similar to what has been observed in $\mathrm{T}_{\mathrm{RM}}$ in the $\operatorname{skin}^{34}$, brain ${ }^{35}$, and lung ${ }^{36}$, and in intrahepatic NK cells ${ }^{10}$. A reduced cytolytic activity in the brain has been postulated to prevent immune pathology in response to low or non-specific stimuli ${ }^{35}$, a similar mechanism could be at play in the liver. On the other hand, as part of the intrahepatic CD69 + CD8 + T cell population consisted of MAIT cells, it cannot be excluded that some of these differences could be caused by this population which is generally low in cytotoxic content ${ }^{37}$. In CHB patients, more liver-resident $\mathrm{T}_{\mathrm{EFF}}$ as well as $\mathrm{T}_{\mathrm{MEM}}$ cells expressing granzyme $\mathrm{B}$ were observed than in $\mathrm{CHC}$ and control patients, possibly antagonizing tolerance and adding to liver damage. The infiltration of a dense non-virus specific $\mathrm{T}$ cell population has previously been suggested to drive liver damage in viral hepatitis ${ }^{9,38}$.

T-bet, Hobit and Eomes are the leading transcription factors thought to govern the process of $\mathrm{T}$ cell differentiation $^{21,22,39-41}$, as well as $\mathrm{T}_{\mathrm{RM}}$ development ${ }^{13,22}$. T-bet and Hobit were expressed by significantly less cells in the intrahepatic CD69+CD8 + T cell population as compared to peripheral blood CD8 + T cells. As these transcription factors play an important role in the regulation of cytolytic protein expression ${ }^{41,42}$, the distinct transcriptional profile observed here possibly induced a reduction in perforin and granzyme B expression. A significantly larger proportion of intrahepatic CD69- CD8+ cells expressed T-bet and Hobit as compared to CD69+ cells, whereas the IFN- $\gamma$ mRNA content did not differ between these populations. This suggests that in the liver, mechanisms additional to T-bet and Hobit are regulators of IFN- $\gamma$ production ${ }^{21,41,43}$. Hobit expression has been shown to be important in the development of liver $\mathrm{T}_{\mathrm{RM}}$ in mice $^{13}$. As Hobit expression was observed in liver CD69+ $\mathrm{T}$ cells, this transcription factor may also be involved in $\mathrm{T}_{\mathrm{RM}}$ development in humans. However, significantly less intrahepatic CD69 + CD8 + T cells expressed Hobit as compared to intrahepatic CD69- T cells and blood CD8+ $\mathrm{T}_{\mathrm{EFF}}$ cells, which are well known to express Hobit ${ }^{21}$. Furthermore, in CHC livers, the proportion of Hobit expressing cells was reduced, which indicates that an alternative differentiation process occurs in these cells.

Previous data on human intrahepatic NK cells did not show differences between healthy donor tissue obtained from organ donors and resection margins of liver metastases ${ }^{10}$. However, it would be interesting to evaluate the phenotype of liver the $\mathrm{T}_{\mathrm{RM}}$ population in truly healthy subjects in further studies. Other groups have already identified the presence of substantial populations of $\mathrm{T}_{\mathrm{RM}}$ in many tissues in organ donors ${ }^{3,7}$. Similar to their results, in this study we have identified an intrahepatic $\mathrm{T}$ cell population with a specific tissue resident phenotype. The presence of a chronic viral infection in the liver seems to infer specific changes to the differentiation status of this intrahepatic CD69 + CD8 + T cell population, and suggests an altered cytotoxic potential in CHB and specific regulation of differentiation and homing capabilities in $\mathrm{CHC}$ infection. Understanding the role of tissue-resident $\mathrm{T}$ cells in chronic viral hepatitis may help the development of future therapeutic interventions enabling the prevention of liver cirrhosis and hepatocellular carcinoma.

\section{Patients and Methods}

Patient samples. We included 17 patients of which 7 did not have viral hepatitis (controls), 4 had chronic HBV (CHB) infection and 6 had chronic HCV (CHC) infection. Patient characteristics are listed in Table 1. The study was approved by the Ethical Review Board of the Academic Medical Center, Amsterdam, The Netherlands and all patients gave written informed consent in accordance with the Declaration of Helsinki. The study was performed in accordance with Good Clinical Practice guidelines, Good Laboratory Practice guidelines and local regulatory requirements. Liver tissue with paired blood samples were obtained from patients undergoing surgical liver resection (indications listed in Table 1). From the resected liver tissue, the non-affected, tumor-free margin surrounding the pathology was obtained. Liver samples were perfused to remove any residual blood, disrupted and treated with collagenase IV (Worthington Biochemical Corporation, CA, USA) and DNAse I (Sigma-Aldrich, MO, USA). Mononuclear cells from blood and liver were isolated using standard density-gradient centrifugation and cryopreserved for later analysis.

Flow cytometry. Cells were thawed and stained with different combinations of fluorescent label-conjugated mouse monoclonal antibodies (listed in the Supplementary Materials). For intracellular staining, cells were fixed after surface staining, permeabilized, and stained with fluorescent label-conjugated mouse monoclonal antibodies (Supplementary Materials). All measurements were done on an LSR Fortessa flowcytometer (BD Biosciences, CA, USA) and analyzed by FlowJoMacV 9.7.5 software (Tree Star Inc., OR, USA). 


\begin{tabular}{|l|l|l|l|l|l|l|l|}
\hline Case\# & Viral status & Gender & Age & Reason surgery & Cirrhosis* & Genotype & $\begin{array}{l}\text { ALT } \\
\text { (U/l) }\end{array}$ \\
\hline 1 & Control & $\mathrm{m}$ & 81 & cholangiocarcinoma & no & & 42 \\
\hline 2 & Control & $\mathrm{m}$ & 81 & liver metastasis CRC & no & & 30 \\
\hline 3 & Control & $\mathrm{f}$ & 50 & liver metastasis CRC & no & & 33 \\
\hline 4 & Control & $\mathrm{m}$ & 68 & HCC & no & & 24 \\
\hline 5 & Control & $\mathrm{f}$ & 37 & multiple adenomas & no & & 50 \\
\hline 6 & Control & $\mathrm{m}$ & 68 & intrahepatic gallstones & no & & 32 \\
\hline 7 & Control & $\mathrm{m}$ & 67 & liver metastasis CRC & no & & 18 \\
\hline 8 & CHB & $\mathrm{m}$ & 51 & HCC & yes & C & 81 \\
\hline 9 & CHB & $\mathrm{m}$ & 57 & HCC & F3/F4 & C & 33 \\
\hline 10 & CHB & $\mathrm{m}$ & 47 & cholangiocarcinoma & F1 & unknown & 43 \\
\hline 11 & CHB & $\mathrm{m}$ & 64 & HCC & F3 & unknown & 11 \\
\hline 12 & CHC $(\mathrm{HBVc})$ & $\mathrm{m}$ & 58 & HCC & yes & lb & 57 \\
\hline 13 & CHC $(\mathrm{HBVc})$ & $\mathrm{m}$ & 58 & HCC & F2/F3 & 1b & 129 \\
\hline 14 & CHC & $\mathrm{m}$ & 59 & HCC & yes & 1a & 154 \\
\hline 15 & CHC & $\mathrm{m}$ & 61 & HCC & yes & $1 \mathrm{a}$ & 111 \\
\hline 16 & CHC & $\mathrm{m}$ & 52 & HCC & yes & $4 \mathrm{a}$ & 30 \\
\hline 17 & CHC & $\mathrm{m}$ & 55 & HCC & 112 \\
\hline
\end{tabular}

Table 1. Patient characteristics. *Cirrhosis based on METAVIR score at histology. Abbreviations; ALT, alanine aminotransferase; m, male; f, female; CRC, colorectal carcinoma; HCC, hepatocellular carcinoma; CHB, chronic hepatitis B; $\mathrm{CHC}$, chronic hepatitis C; $\mathrm{HBV}$, cleared acute hepatitis B virus.

Immunohistochemistry. Paraffin embedded formalin-fixed sections $(4 \mu \mathrm{m})$ of non-affected liver tissue from control patients $(n=3)$ were stained using sequential immunohistochemistry ${ }^{44}$. Sections were deparaffinized and rehydrated in up to $96 \%$ ethanol. Endogenous peroxidase activity was blocked by incubation with $0.3 \%$ $\mathrm{H} 2 \mathrm{O} 2$ in methanol. Antigen retrieval was performed at $120^{\circ} \mathrm{C}$ under pressure in a Tris/EDTA buffer. The sections were stained overnight with anti-CD69 (mouse IgG1, clone CH11, Abcam, Cambridge, UK), followed by incubation with polyHRP-anti-mouse IgG (ImmunoLogic, Duiven, Netherlands). HRP activity was demonstrated with VectorNovaRed (Vector Laboratories, CA, USA). The slides were counterstained with hematoxilin, coverslipped and scanned in a Philips Ultra Fast Scanner 1.6RA (Philips, Eindhoven, Netherlands). After removal of the coverslip, the sections were stripped in a Tris-SDS buffer at $50^{\circ} \mathrm{C}$. Next, they were incubated with anti-CD103 (rabbit IgG, clone EPR4166, Abcam, Cambridge, UK) and subsequent polyHRP-anti-rabbit IgG (ImmunoLogic, Duiven, Netherlands). HRP activity was demonstrated as above. This procedure was repeated with anti-CD8 (mouse IgG1, clone C3/144B, Dako, Glostrup, Denmark) and anti-CD3 (rabbit IgG1, clone Sp7, Thermo scientific, NH, USA). Images were analysed with Fiji Image J software ${ }^{45}$. Pseudocolors were applied to provide co-localization images.

Cell sorting and RNA extraction. Liver lymphocytes from 6 control patients were sorted using fluorescent label-conjugated mouse monoclonal antibodies for CD3, CD8 and CD69 (Supplementary Materials). Liver CD3 + CD8+ lymphocytes were sorted into a CD69+ and CD69- fraction on a Sony SH800 cell sorter (Sony biotechnology Inc., CA, USA). After sorting, RNA was extracted with the AllPrep isolation kit (Qiagen, CA, USA).

Reverse transcription quantitative polymerase chain reaction (RT-qPCR). Reverse transcription of mRNA was performed using M-MLV reverse transcriptase and oligo-dT primers. Relative quantification of gene expression was determined with the LightCycler 2.0 or LightCycler 480 Real-Time PCR System (Roche Applied Science, CT, USA) using the SYBR Green PCR Master Mix and gene specific primers (Supplementary Table 1). mRNA expression levels were normalized to the arithmetic mean of the housekeeping gene ACTB using the comparative Ct method, and $\log 10$ transformed for analysis.

Data availability. The datasets generated during and/or analysed during the current study are available from the corresponding author on reasonable request.

Statistical analyses. Statistical analyses were performed using GraphPad Prism 6 software (GraphPad Software Inc., CA, USA). For paired data, the paired t-test (Gaussion distribution) or Wilcoxon signed rank test (non-Gaussion distribution) was used. Similarly, for non-paired data, the t-test or Mann-Whitney U test was used. Correlations were calculated using the Spearman rank test. Two sided p-values below 0.05 were considered to be statistically significant.

\section{References}

1. Knolle, P. A. \& Thimme, R. Hepatic immune regulation and its involvement in viral hepatitis infection. Gastroenterology 146, 1193-1207 (2014).

2. Hamann, D. et al. Phenotypic and functional separation of memory and effector human CD8+ T cells. J. Exp. Med. 186, 1407-1418 (1997).

3. Sathaliyawala, T. et al. Distribution and Compartmentalization of Human Circulating and Tissue-Resident Memory T Cell Subsets. Immunity 38, 187-197 (2013) 
4. Watanabe, R. et al. Human skin is protected by four functionally and phenotypically discrete populations of resident and recirculating memory T cells. Sci. Transl. Med. 7, 279ra39 (2015).

5. Park, C. O. \& Kupper, T. S. The emerging role of resident memory T cells in protective immunity and inflammatory disease. Nat. Med. 21, 688-97 (2015).

6. Farber, D. L., Yudanin, N. A. \& Restifo, N. P. Human memory T cells: generation, compartmentalization and homeostasis. Nat. Rev. Immunol. 14, 24-35 (2014).

7. Thome, J. J. C. et al. Spatial Map of Human T Cell Compartmentalization and Maintenance over Decades of Life. Cell 159, 814-828 (2014).

8. Morrot, A., Hafalla, J. C. R., Cockburn, I. A., Carvalho, L. H. \& Zavala, F. IL-4 receptor expression on CD8+ T cells is required for the development of protective memory responses against liver stages of malaria parasites. J. Exp. Med. 202, 551-560 (2005).

9. Abrignani, S. Bystander activation by cytokines of intrahepatic T cells in chronic viral hepatitis. Semin. Liver Dis. 17, 319-22 (1997).

10. Stegmann, K. A. et al. CXCR6 marks a novel subset of T-bet lo Eomes hi natural killer cells residing in human liver. Sci. Rep. 6, 26157 (2016).

11. Skon, C. N. et al. Transcriptional downregulation of S1pr1 is required for the establishment of resident memory CD8+ T cells. Nat. Immunol. 14, 1285-93 (2013).

12. Shiow, L. R. et al. CD69 acts downstream of interferon-alpha/beta to inhibit S1P1 and lymphocyte egress from lymphoid organs. Nature 440, 540-544 (2006).

13. Mackay, L. K. et al. Hobit and Blimp1 instruct a universal transcriptional program of tissue residency in lymphocytes. Science. 352, 459-63 (2016).

14. Heydtmann, M. et al. CXC chemokine ligand 16 promotes integrin-mediated adhesion of liver-infiltrating lymphocytes to cholangiocytes and hepatocytes within the inflamed human liver. J. Immunol. 174, 1055-1062 (2005).

15. Tse, S. W., Radtke, A. J., Espinosa, D. A., Cockburn, I. A. \& Zavala, F. The chemokine receptor CXCR6 is required for the maintenance of liver memory $\mathrm{CD} 8^{+} \mathrm{T}$ cells specific for infectious pathogens. J. Infect. Dis. 210, 1508-1516 (2014).

16. Reantragoon, R. et al. Antigen-loaded MR1 tetramers define $\mathrm{T}$ cell receptor heterogeneity in mucosal-associated invariant $\mathrm{T}$ cells. J. Exp. Med. 210, 2305-2320 (2013).

17. Moran, A. E. et al. T cell receptor signal strength in Treg and iNKT cell development demonstrated by a novel fluorescent reporter mouse. J Exp Med 208, 1279-1289 (2011).

18. Appay, V., Van Lier, R. A. W., Sallusto, F. \& Roederer, M. Phenotype and function of human T lymphocyte subsets: Consensus and issues. Cytom. Part A 73, 975-983 (2008).

19. Gupta, S. \& Gollapudi, S. Effector Memory CD8+ T Cells Are Resistant to Apoptosis. Ann. N.Y. Acad. Sci. 1109, 145-150 (2007).

20. Smith, C. et al. Endogenous antigen presentation impacts on T-box transcription factor expression and functional maturation of CD8+ T cells. Blood 120, 3237-3245 (2012).

21. Vieira Braga, F. A. et al. Blimp-1 homolog Hobit identifies effector-type lymphocytes in humans. Eur. J. Immunol. 45, 2945-2958 (2015).

22. Mackay, L. K. et al. T-box Transcription Factors Combine with the Cytokines TGF- b and IL-15 to Control Tissue-Resident Memory T Cell Fate. Immunity 43, 1101-1111 (2015).

23. Steinert, E. M. et al. Quantifying Memory CD8 T Cells Reveals Regionalization of Immunosurveillance. Cell 161, 737-749 (2015).

24. Casey, K. A. et al. Antigen-Independent Differentiation and Maintenance of Effector-like Resident Memory T Cells in Tissues. J. Immunol. 188, 4866-4875 (2012).

25. Shimizu, Y. et al. Preferential accumulation of CD103+ T cells in human livers; its association with extrathymic T cells. J. Hepatol. 39, 918-924 (2003).

26. Ledgerwood, L. G. et al. The sphingosine 1-phosphate receptor 1 causes tissue retention by inhibiting the entry of peripheral tissue T lymphocytes into afferent lymphatics. Nat. Immunol. 9, 42-53 (2008).

27. Tse, S.-W., Cockburn, I. A., Zhang, H., Scott, A. L. \& Zavala, F. Unique transcriptional profile of liver-resident memory CD8+ T cells induced by immunization with malaria sporozoites. Genes Immun. 14, 302-9 (2013).

28. Geissmann, F. et al. Intravascular immune surveillance by CXCR6 + NKT cells patrolling liver sinusoids. PLoS Biol. 3, e113 (2005).

29. Weninger, W., Crowley, M. A., Manjunath, N. \& von Andrian, U. H. Migratory properties of naive, effector, and memory CD8(+) T cells. J. Exp. Med. 194, 953-966 (2001).

30. Mackay, C. R., Marston, W. L. \& Dudler, L. Naive and memory T cells show distinct pathways of lymphocyte recirculation. J. Exp. Med. 171, 801-17 (1990).

31. de Bree, G. J. et al. Selective accumulation of differentiated CD8+ T cells specific for respiratory viruses in the human lung. J. Exp. Med. 202, 1433-42 (2005).

32. Wakim, L. M. et al. The molecular signature of tissue resident memory CD8 T cells isolated from the brain. J. Immunol. 189, 3462-71 (2012).

33. Crispe, I. N., Dao, T., Klugewitz, K., Mehal, W. Z. \& Metz, D. P. The liver as a site of T-cell apoptosis: graveyard, or killing field? Immunol. Rev. 174, 47-62 (2000).

34. Schaerli, P. et al. A skin-selective homing mechanism for human immune surveillance T cells. J. Exp. Med. 199, 1265-1275 (2004).

35. Smolders, J. et al. Characteristics of differentiated CD8+ and CD4+ T cells present in the human brain. Acta Neuropathol. 126, 525-535 (2013).

36. Piet, B. et al. $\mathrm{CD} 8^{+} \mathrm{T}$ cells with an intraepithelial phenotype upregulate cytotoxic function upon influenza infection in human lung. J. Clin. Invest. 121, 2254-63 (2011).

37. Kurioka, A., Walker, L. J., Klenerman, P. \& Willberg, C. B. MAIT cells: new guardians of the liver. Clin. Transl. Immunol. 6, e132 (2017).

38. Maini, M. K. et al. The role of virus-specific $\mathrm{CD} 8(+)$ cells in liver damage and viral control during persistent hepatitis B virus infection. J. Exp. Med. 191, 1269-80 (2000).

39. Hersperger, A. R. et al. Increased HIV-specific CD8+ T-cell cytotoxic potential in HIV elite controllers is associated withT-bet expression. Blood 117, 3799-3809 (2011).

40. Intlekofer, A. M. et al. Effector and memory CD8+ T cell fate coupled by T-bet and eomesodermin. Nat. Immunol. 6, 1236-1244 (2005).

41. Pearce, E. L. et al. Control of effector CD8+ T cell function by the transcription factor Eomesodermin. Science. 302, 1041-1043 (2003).

42. van Gisbergen, K. P. J. M. et al. Mouse Hobit is a homolog of the transcriptional repressor Blimp-1 that regulates NKT cell effector differentiation. Nat. Immunol. 13, 864-871 (2012).

43. Hombrink, P. et al. Programs for the persistence, vigilance and control of human CD8+ lung-resident memory T cells. Nat Immunol $17,1467-1478(2016)$

44. van den Brand, M. et al. Sequential immunohistochemistry: A promising new tool for the pathology laboratory. Histopathology 65, 651-657 (2014)

45. Schindelin, J. et al. Fiji: an open-source platform for biological-image analysis. Nat. Methods 9, 676-682 (2012). 


\section{Acknowledgements}

The authors would like to thank Dr. L. Swadling, Dr. K.M.H. Heutinck and Prof. Dr. C.P. Engelfriet for critically revising the manuscript. In addition, the authors thank N. Claessen for assistance with immunohistochemistry, Dr. E.B.M. Remmerswaal and Ing. B. Hooibrink for technical assistance with FACS and D.S. Koenis for kindly providing Nur77 primers. This work was supported by the Dutch Society for Gastroenterology and Hepatology.

\section{Author Contributions}

F.S. and A.d.N. designed the study and included patients. F.S., M.J.S. and K.A.v.D. performed the analyses. F.S. wrote the manuscript. A.d.N., M.J.S., K.A.v.D., K.P.J.M.v.G., J.V., E. M.M.v.L., H.W.R. and N.A.K. critically revised the manuscript.

\section{Additional Information}

Supplementary information accompanies this paper at doi:10.1038/s41598-017-06352-3

Competing Interests: Hendrik W. Reesink serves as a consultant for AbbVie, Alnylam, Bristol Myers Squibb, Gilead Sciences, Janssen-Cilag, Merck, PRA International, Regulus, Replicor, Roche and R-Pharm and received grant/research support from AbbVie, Bristol Myers Squibb, Boehringer Ingelheim, Gilead Sciences, JanssenCilag, Merck, PRA International, Regulus, Replicor and Roche. All other authors: None declared.

Publisher's note: Springer Nature remains neutral with regard to jurisdictional claims in published maps and institutional affiliations.

(c) Open Access This article is licensed under a Creative Commons Attribution 4.0 International

License, which permits use, sharing, adaptation, distribution and reproduction in any medium or format, as long as you give appropriate credit to the original author(s) and the source, provide a link to the Creative Commons license, and indicate if changes were made. The images or other third party material in this article are included in the article's Creative Commons license, unless indicated otherwise in a credit line to the material. If material is not included in the article's Creative Commons license and your intended use is not permitted by statutory regulation or exceeds the permitted use, you will need to obtain permission directly from the copyright holder. To view a copy of this license, visit http://creativecommons.org/licenses/by/4.0/.

(C) The Author(s) 2017 